\title{
Development of Earthquake Early Warning System Using ADXL335 Accelerometer
}

\author{
Yoga Priyana ${ }^{1}$, Folkes Laumal ${ }^{2}$, Emir Husni $^{3}$ \\ ${ }^{1,3}$ School of Electrical Engineering \& Informatics, Institut Teknologi Bandung, Bandung 40132, Indonesia \\ ${ }^{2}$ Electrical Engineering, Politeknik Negeri Kupang, Kupang, Indonesia
}

\begin{abstract}
Indonesia is an archipelago located at three earthquake belts. This condition cause an earthquake can occur anytime and threaten human life. A quick and accurate early warning system by using the seismic wave data processing is required so, the number of victims affected by the earthquake can be shortened. Here, ADXL335 accelerometers are used as seismic sensors with an Arduino minimum system. The results show that when the first earthquake's vibration occurs, $\mathrm{P}$ wave data detected by the ADXL335 sensor is successfully buffered, calibrated, transmitted and displayed on the server. When there are errors on the transmission, server will request for retransmission. The alarm of the earthquake early warning system will be activated if there are at least three sensors from different locations successfully transmit $\mathrm{P}$ wave data with the same scale. This is needed to prevent fake seismic waves.
\end{abstract}

Keywords: Early warning system, earthquake, ADXL335.

\section{INTRODUCTION}

Indonesia is a confluence of three major tectonic plates, there is an Indo-Australian, Eurasian and Pacific. Around the meeting point is the accumulation of plate collision energy and when the layer of earth not able to hold energy anymore, it will release during energy named earthquake (Primary wave and Secondary wave). The experience of the earthquake in some areas has provided thousands of lives and properties, so its necessary to measures of mitigation to reduce the earthquake risk. In short time when the earthquake happens, can be used to provide early warning to the community through the development of early warning system based on the arrival of the $\mathrm{P}$ wave, the first wave that arrives before the real earthquake happens ( $\mathrm{S}$ wave).

"Email Address: yoga@1skk.ee.itb.ac.id

An earthquake early warning is personalized in alarm and running text that executed on a sensor system. Preparedness and speed of response are necessary because of narrow time from passing information to alleged actual of arrival earthquake. The earlier information given, the more loose the time for the population to respond.

Currently, Indonesia has seismograph earthquake sensors. Seismograph has sensitive instruments that can detect seismic waves generated by earthquakes. Seismic waves that occurred during the earthquake displayed as a wavy line on the seismogram.

With the evolution of IC technology, many microcontroller are produced. The microcontroller is equipped with internal memory blocks, accumulator, ALU, RAM, I/O ports and interrupt ports that support package as a medium for reading, processing and writing data from and to other components, so there are some microcontroller-based sensor systems being made with the more complete working principle ${ }^{1}$. One of a circuit integrated developed with microcontroller is Arduino. 
There are three types of accelerometers: a single axis acceleration to detect movement of an object in one direction, 2-axis acceleration to detect object motion in 2directions and 3-axis acceleration to detect motion 3directions.

ADXL is 3 -axis accelerometer sensor with 3.3 volt analog input. In that respect are several series of these accelerometers, including ADXL330, ADXL335 and ADXL345. The accelerometer has $\pm 3 \mathrm{~g}$ sensitivity of each axis $(\mathrm{x}, \mathrm{y}, \mathrm{z})$ and can be used to measure the static and dynamic acceleration, object motion, collisions and vibrations $\mathrm{s}^{3,4}$.

This research aims to develop an earthquake early warning system using ADXL335 accelerometer through several problems, namely:

1. How to design an earthquake early warning system using the ADXL335 accelerometer,

2. How to make calibration of ADXL335 in order to obtain the reference as an indicator of the vibration occurrence,

3. How to make transmission of data from sensor to client and client to server using communication medium,

4. How to handle loss/error occurred when data sent to the server,

5. How to declare the earthquake early warning.

The research is expected to contribute in seismology for giving early warning when an earthquake will come, machinery vibration analysis, object tilt and collision analysis.

\section{SURVEY AND METHOD}

\subsection{Early warning system}

The Early Warning System is a series of systems to inform the public about the emergence of a natural event (natural signs). Early warning of disaster was act of providing information in communicating with easily understood language. In critical condition, manifested in the form of an early warning is alarming. The earlier information submitted, people experience more time to respond and rescue of their lifetimes.

Early warning information obtained in two ways, conventional and modern. Conventionally, disastrous introduction is done by the introduction of natural phenomena that prior to the disaster, which is tailored to the characteristics of disaster. Modern ways, disastrous introduction are done with the monitoring atmosphere activity periodically by satellite or high-tech equipment. People who living in prone areas should be empowered and respond to natural disasters toll system so that number of victims can be slimmed down ${ }^{5}$.

\subsection{Seismic waves}

Seismic waves are waves that propagate inside or surface on earth from seismic sources such as from annearthquakee. Volcanic eruptions, landslides, storms and pounding supersonic aircraft. There are two types of seismic waves, namely Primary waves $(\mathrm{P})$ and Secondary Waves (S). Primary wave that occurs when the initial vibrations occur and propagate as longitudinally. Secondary wave propagates like as a wave of water that moves up and down with speed about $4 \mathrm{~km} / \mathrm{Sec}$ and slower than Primary. Secondary wave has a large amplitude so it has the power to destroy buildings or cliffs $^{6}$.

\subsection{UDP Socket}

UDP socket is the TCP / IP protocol that connectionless and unreliable. Connectionless meaning that data sent without to negotiate between two hosts and unreliable meaning that the message sent without accompanied by an acknowledgment. Application layer protocol that works with UDP socket must perform the recovery of the message that is lost during transmission ${ }^{7,8}$. UDP have 32 bit data format as indicated in Figure 1 with maximum data transfer is $65 \mathrm{~KB}$ every time send.

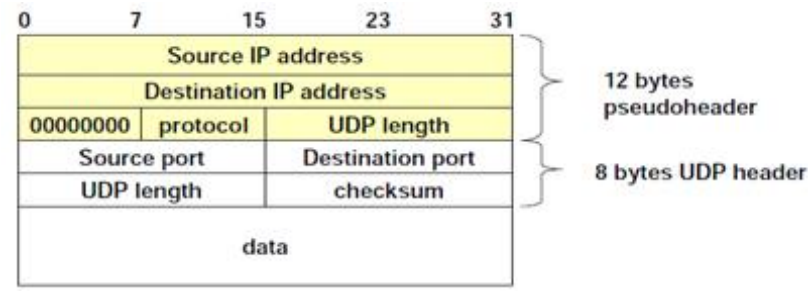

Fig.1. UDP Data Format ${ }^{7}$

\section{DESIGN}

In general, early warning system in this research provides information based on the data primary waves that first received by the system so that rescue efforts can be done as early before the actual earthquake arrives ( $\mathrm{S}$ wave). $\mathrm{P}$ waves propagate in longitudinal and $\mathrm{S}$ wave in transfersal. Arrival times of $S$ wave typically ranging from seconds to minutes depending on sensor distance from the earthquake epicenter ${ }^{6}$.

Here, the design of earthquake early warning system divided in two parts, the Base Station and the Server. In the both parts, there are 2 users with an identity and responsibility for observing data processing. Specifically, in the server, the user has an authorized to make sure the truth of early warning.

The Base Station consists of 2 parts and placed near to the earthquake points, namely:

1) Seismic sensors which are placed against on the cement floor and consists of Arduino as minimum system, ADXL335 as vibration sensor for detects vibration in 3 axes $^{3}$ and EM411 as a position sensor for detects the distance from GPS satellites and convert it using the NMEA 0183 standard $^{9}$. All of that are connected through I/O pins on each element. On the Arduino equipped with 32 KB ATmega328 microcontroller, the block amplifier and ADC to strengthen and data convert.

2) Client with a complete database system to 
accommodate all sensor data send by an Arduino. Communication from earthquake sensor to client using 1.5 meters of USB cable.

The design of Base Station can be seen in Figure 2.

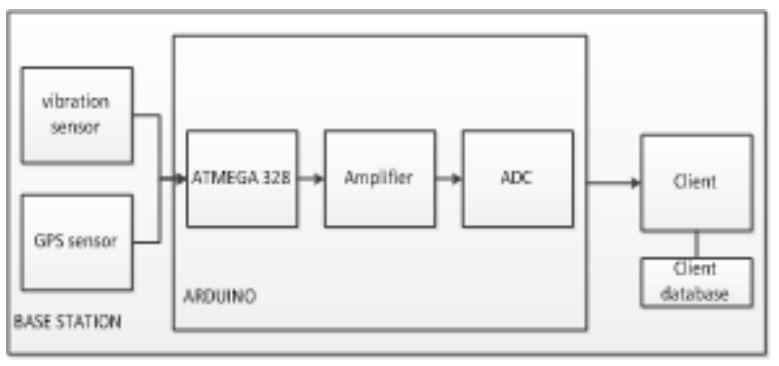

Fig.2. Design of Base Station

The client then connected to server using 13.5 Mbps max internet connection without the barrier. Location of server placed in the government building that handles of the seismic. The server also has a database system that will accommodate data from all clients that connected to it. Communication client to server using UDP socket to accelerate data transmission. Format data applied to the system are 32 bits because the architecture of microcontroller supports of this format.

Full design of the sensor system is shown in Figure 3.

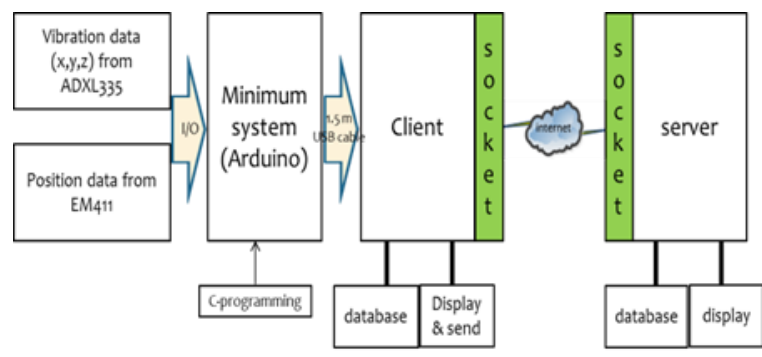

Fig.3. Full Design of Earthquake Sensor

Design of earthquake sensor system consists of hardware and software. Hardware design includes of interconnection sensor to Arduino, Arduino to client and client to server.

The design of software includes of communication data sensors (AXL335, EM411) to Arduino, Arduino to the client and node to server. Communication sensor to Arduino as in Figure 4.

There are 2 data taken from sensor, namely position data from EM411 and vibration data (x,y,z) from ADXL335. Foremost, the Arduino will taken position data and putting into port and second, Arduino has taken vibration data $(\mathrm{x}, \mathrm{y}, \mathrm{z})$ and store in buffer for calibrating.

The Arduino will calibrate data in buffer with specified zero_G and scale values. Zero_G is the axis value when the sensor is in a stationary position without reversed and the scale is the average of change in each axis. Based on calculations, the value of zero_G are 384, 383 and 473 for each axis and scale is 102.3 . One vector formula used to generate $\mathrm{x}, \mathrm{y}, \mathrm{z}$ voltage and Acceleration $(\mathrm{A})^{10,11}$.

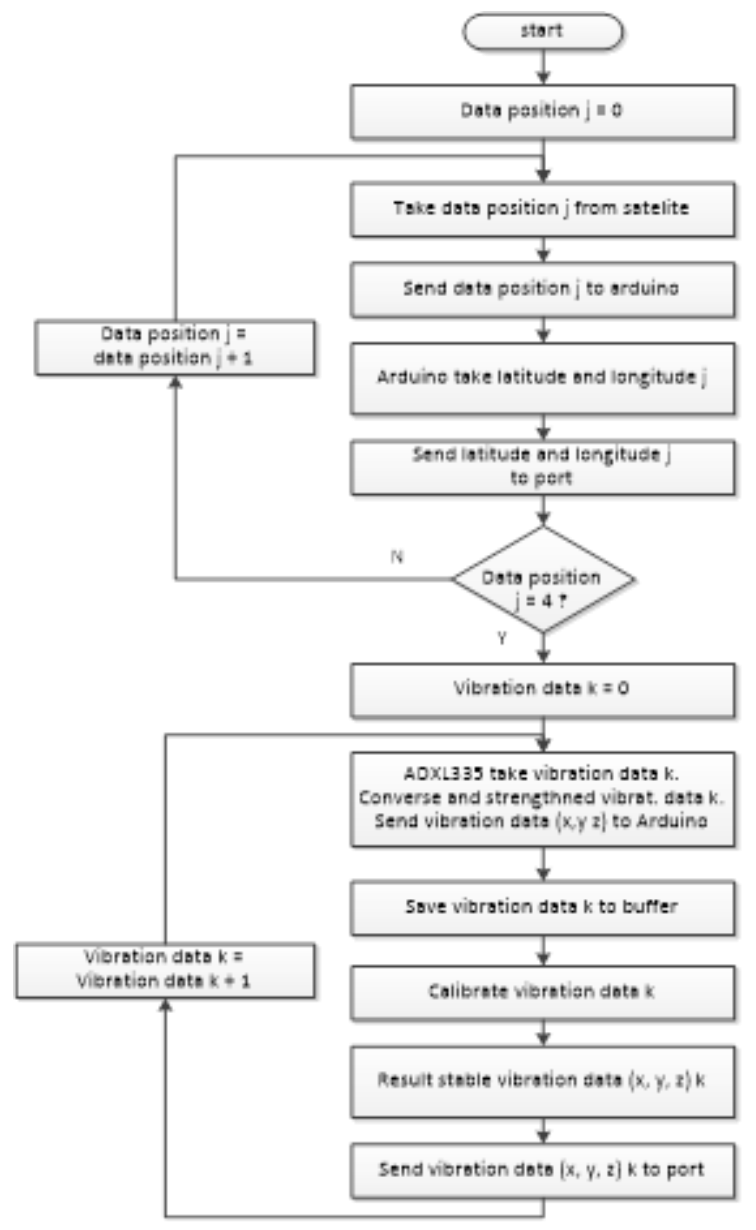

Fig.4. Communication Sensor to Arduino

From calculations, If there is no vibration, ADXL335 gives the voltage value $0.000,0.000,0.000$ for $\mathrm{x}$-axis, $\mathrm{y}$-axis and $\mathrm{z}$-axis and Acceleration is 0.000 . $\mathrm{Vx}$ _axis, Vy_axis and $\mathrm{Vz}$ _axis values, then used as reference for chart display, $\mathrm{Vy}_{-}$axis value and its changes as a reference for determining scale whenever an earthquake occurred. When no vibration, 3-axis voltage value will stabilize at the calibration value. But when trigger given (vibration), the voltage value will be changed.

A communication client - server designed with UDP socket protocol in MYSQL. The selection of this protocol because it is faster, does not require back and a handshake ${ }^{7}$.

Data send as serial start with latitude, longitude, $\mathrm{x}$ axis, y-axis and z-axis with maximum data 216-1 - 20 byte header -8 byte UDP header $=65535$ bytes $(65 \mathrm{~KB})$ every transmitter. Identity of client and server using local IP with port 3306 which support mysql.

Handling of loss/error data is designed in two phases: detection and correction error. Detection phase performed with comparing vibration data and its duplicate. If it is same, the true status will appear on the web. Only if one is empty, and so the condition of loss data appears. Correction phase is performed by requiring the client to 
resend the correct information.

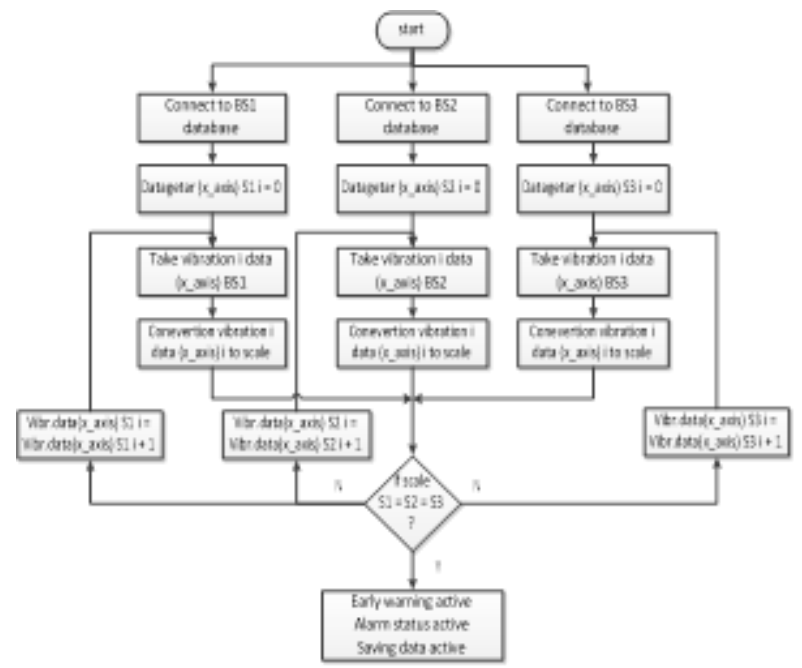

Fig.5. Determine earthquake early warning

Figure 5 shows the determining of early warning status. The server need 3 vibration values from the 3 sensors at different locations. Each sensor will sends vibration data ( $\mathrm{x}$-axis, $\mathrm{y}$-axis, $\mathrm{z}$-axis) to database and server will check and match the first vibration data on a specified range of scale. If all sensors give the same scale, the server will declare the earthquake early warning and activate of alarm. This method is used as avoiding a fake signals.

Scales that used to determine of an early warning system as indicated in Table 1.

Table.1. Conversion of 3-axis value from Zerro_G to Scale.

\begin{tabular}{|l|l|l|l|}
\hline $\begin{array}{l}\text { Before } \\
\text { calibration }\end{array}$ & $\begin{array}{l}\text { After } \\
\text { calibration }\end{array}$ & $\begin{array}{l}\text { Multiply } \\
\text { factor }\end{array}$ & $\begin{array}{l}\text { End Scale } \\
\text { in use }\end{array}$ \\
\hline $383-390$ & $0.000-0.010$ & 100 & 1 \\
\hline $390-397$ & $0.010-0.020$ & 100 & 2 \\
\hline $397-405$ & $0.020-0.030$ & 100 & 3 \\
\hline $405-412$ & $0.030-0.040$ & 100 & 4 \\
\hline $412-419$ & $0.040-0.050$ & 100 & 5 \\
\hline $419-426$ & $0.050-0.060$ & 100 & 6 \\
\hline $426-433$ & $0.060-0.070$ & 100 & 7 \\
\hline $433-440$ & $0.070-0.080$ & 100 & 8 \\
\hline $440-448$ & $0.080-0.090$ & 100 & 9 \\
\hline $448-455$ & $0.090-0.100$ & 100 & 10 \\
\hline $455-463$ & $0.100-0.110$ & 100 & 11 \\
\hline $463-473$ & $0.110-0.120$ & 100 & 12 \\
\hline
\end{tabular}

\section{RESULT AND DISCUSSION}

With 1.5 meters USB cable, the earthquake sensor will connect to the client. After the client turn on, the internet Wifi will be active. The earthquake sensor connecting with other devices is shown in Figure 6 and Figure 7.

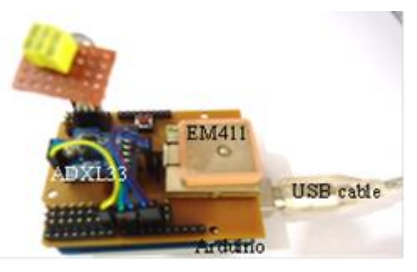

Fig.6. Earthquake Sensor.

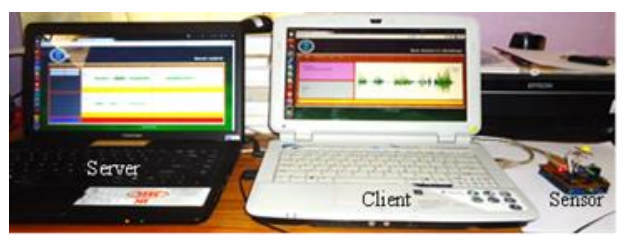

Fig.7. Sensor, Client and Server Connections

Data from the sensor are sent to the Arduino every 0.01 seconds. The information will be sent to buffer for calibration. Three axis calibration results show that vibration values are not always stable when no vibration condition $(0.000,0.000,0.000)$. Sometimes changes to 0.010 or -0.010 . But from characteristics of ADXL335, Noise Density founded for Xaxis, Yaxis at $150 \mu \mathrm{m}$ and Zaxis at $300 \mu \mathrm{m}^{3}$, so Peak Noise Density for Xaxis and Yaxis are 0.084 and 0.064 for Zaxis. This result is still under Peak Noise Density. When there is no vibration, data is not taken.

The next test is shaking the sensor to ensure the communication process among sensors, Arduino, client and server are correct and graphs vibration appears. The result shows that vibration data are successful sent to client every 0.01 second and protocol functions is successful. Next, the first vibrations of data successfully send to the server every 0.01 second and displayed in a graph as Figure 8 and 9

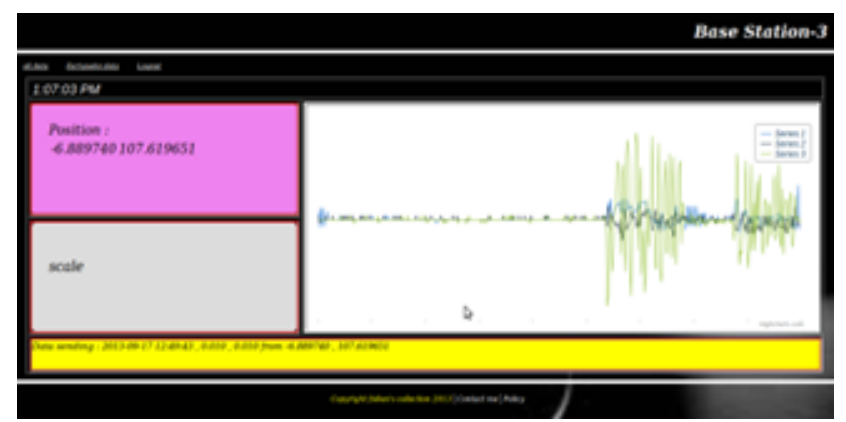

Fig.8. Vibration display in the client 


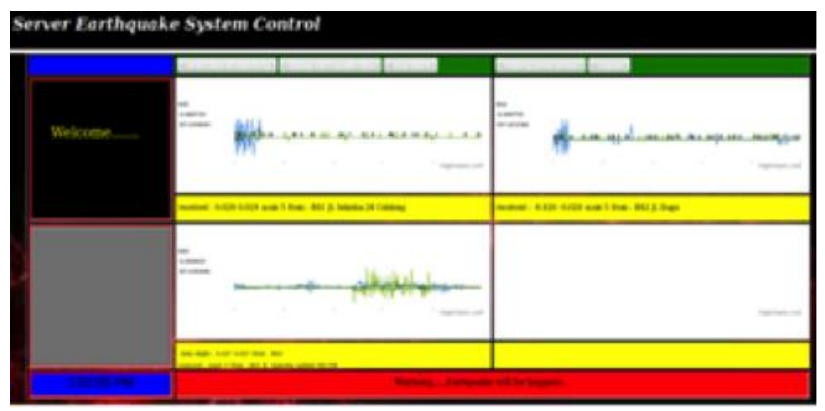

Fig.9. Vibration display in the server

If the loss/error data event is occurred, the server will display the loss status on the web and will be repaired after client resend the correct data.

On the server, status of earthquake early warning is active with the reference of the first vibration data from 3 different base stations. First vibrations data is P-wafe data. This data will be stored in the database. The server does not directly declare the warning status because it still have to wait for the arrival of the P-wave data from 2 base stations. There were 3 seconds time delay given to adjusting the data to other sensors. If 2 other sensor data show the same P-wave value with the same scale, then the server will give early warning and activate the alarm. But if one of the sensors is different, then the server assumes nothing happened. Total time for sending the first vibration data from the sensor to the server and alarm activated is $0.01 \mathrm{~s}+0.01 \mathrm{~s}+0.01 \mathrm{~s}+3 \mathrm{~s}=3.03 \mathrm{~s}$.

Location of all sensors can be viewed through the map. When the sensors indicate warning, the marker will be red, but will be green if nothing happened. Display of sensor location on the map is shown in Figure 10.

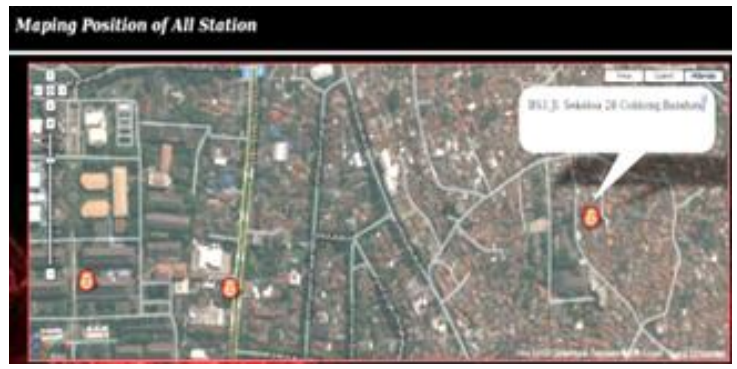

Fig.10. Mapping Status of Sensor

\section{CONCLUSION}

Development of earthquake early warning system can be made with the ADXL335 accelerometer and an Arduino UNO system. The first vibration data can act as reference of Primary wave. Calibration process is done by taking the 3-axis value of ADXL335 when no vibration condition. The 3-axis value is processed with other variables in the database. Communication from the sensor to the client is performed by the USB cable and from the client to the server by internet using the UDP protocol. Handling of loss/error is done with detection and correction. Error detection is done by duplicating data before sending and error correction by asking the client to resend the correct data. An earthquake early warning will be declared if there are 3 Base Stations (sensors) sending first vibration data ( $\mathrm{P}$ wave) with the sama scale.

When first vibrations detected, this system only needs 3.03 secs to activate early warning status and make alarm ON. This time is measured in conditions as follows: connection sensor to the client using 1.5 meter USB cable, connecting client to server using $13.5 \mathrm{Mbps}$ speed Internet network.

\section{REFERENCES}

[1] Raj Kamal, Microcontrollers: Architecture, Programming, Interfacing and System Design, $2^{\text {nd }}$ Ed., India : Pearson Education, 2011.

[2] Ashish G.Bajaj and Vikran R. Bajaj, Build IEEE 802.15.4 Wireless Sensor Network for Emergency Response Notification for Indoor Situations, International Journal of Engineering Trend in Electrical and Electronics (IJETEE), vol. 1, Issue. 2, March. 2013.

[3] Small, Low Power, 3-Axis $\pm 3 g$ Accelerometer, ADXL335, Norwood, MA 02062-9106, USA : Analog Devices, Inc. All rights reserved, 2009.

[4] Jonathan Bernstein, Low-Noise MEMS Vibration Sensor for Geophysical Applications, Journal of Microelectromechanical System., vol. 8, Dec. 1999.

[5] Sarwidi, Upaya Pengurangan Dampak Bencana Gempa Bumi, Lokakarya Nasional : Pengembangan Sistem Peringatan Dini, PSBA UGM-PMI Pusat, Yogyakarta, 2001.

[6] Afnimar, Seismologi, edisi pertama, Laboratorium Seismologi Institut Teknologi Bandung, ITB, 2009

[7] Behrouz A. Forouzan, DeAnza College with Sophia Chung Fegan, Data Communication and Networking, $4^{\text {th }}$ Ed. , Mc Graw Hill Higher Education, 2007.

[8] Rajkumar Buyya, Thamarai Selvi Somasundaram and Xingchen Chu, Object Oriented Programming with Java Essentials and Applications, New Delhi, India : McGraw-Hill Education (India) Pvt Ltd.

, EM411 GPS Receiver Engine Board, Globalsat Technology Corporation, Doc Version 1.2.

[10] Frederic Camps, Sebastian Harase and Andre Monin, Numerical Calibration for 3-axis Accelerometers and Magnetometer, CNRS/LAAS-University of Tolouse-France, IEEE., 978-1-42443355-1, Sept. 2009.

[11] Tadej Bwravs and Others, Three-Axial Accelerometer Calibration using Kalman Filter Covariance Matrix for Online Estimation of Optimal Sensor Orientation, IEEE., 0018-9456, 2012. 